\title{
Bactericidal Efficacy of Dielectric Barrier Discharge Plasma on Methicillin-Resistant Staphylococcus aureus and Escherichia coli in Planktonic Phase and Colonies In vitro
}

\author{
Niloofar Sanaei, ${ }^{1}$ Halim Ayan ${ }^{1,2, *}$ \\ ${ }^{1}$ Department of Bioengineering, College of Engineering, University of Toledo, Toledo, $\mathrm{OH}, \mathrm{USA}$; \\ 2Department of Mechanical, Industrial, and Manufacturing Engineering, College of Engineering, \\ University of Toledo, Toledo, $\mathrm{OH}$, USA \\ *Address all correspondence to: Halim Ayan, University of Toledo, 5051 Nitschke Hall, Toledo, OH; Tel.: +0014195308126. \\ Fax:+0014195308030. E-mail: Halim.Ayan@utoledo.edu
}

\begin{abstract}
Increasing emergence of antibiotic resistance in bacteria during the past few decades has become a major public health concern and has reduced the efficacy of conventional therapies. The objective of the current study is to examine the bactericidal efficacy of atmospheric pressure nonthermal dielectric barrier discharge (DBD) plasma on methicillin-resistant Staphylococcus aureus (MRSA) as a common musculoskeletal pathogen and Escherichia coli (E. coli) as another cause of bone, joint, and soft tissue infections in planktonic phase and in colonies. In planktonic phase, results demonstrated complete decontamination of E. coli and MRSA cells on agar plates with a bacterial surface density of $1.27 \times 10^{5}$ colony-forming units (CFU)/ $\mathrm{cm}^{2}$ following 30 seconds and 60 seconds of plasma treatment, respectively. Reduction rates of $7 \log _{10}$ steps in the number of viable CFUs in E. coli colonies was observed following 5 minutes of plasma treatment. The colony growth was halted, and no more growth in colony size was observed during a 24-hour monitoring period. For MRSA colonies, results demonstrated $2 \log _{10}$ to $7 \log _{10}$ steps reduction in the number of viable CFUs in each colony after 5 minutes of plasma treatment. Results indicated that plasma-treated colonies will have prolonged lag time during generation of the growth curves. Finally, diffusion of nitric oxide into the agarose gel was confirmed as a reactive agent that was effective in the decontamination process.
\end{abstract}

KEY WORDS: plasma, DBD, sterilization, decontamination, MRSA, E. coli, colony

\section{INTRODUCTION}

Emergence of antibiotic-resistant bacteria is an increasing concern in wound infections. These organisms colonize wounds, often necessitating aggressive wound management with a very limited choice of antibiotics. Staphylococcus aureus is one of the common musculoskeletal pathogens causing osteomyelitis. An infection in bone, cartilage, and surrounding tissues as a result of injury (open wounds) or surgery combined with antibiotic resistance to $S$. aureus is a serious problem..$^{1-4}$

Several conventional techniques are used for the prevention and management of wound infection. It is nonetheless a gold standard to treat patients with deep wounds 
with antibiotic prophylaxis to prevent bacteria from being released into the blood stream and cause infection complications. This procedure is combined with debridement and surgical processes. Washing wounds with antibiotic solutions and use of topical antibiotics are controversial. ${ }^{5}$ In some cases, silver and other noble metals are potential allies in fighting pathogens in wounds. ${ }^{6}$ Considering that antibiotics do not reach the source of the wound infection, only the area around the wound, and that bacteria are becoming resistant to antibiotics, these methods are not very efficient in the treatment of antibioticresistant species. Thus, there is considerable need for an efficient and nondestructive way to decontaminate wounds and prevent germs from spreading. This need fuels the search for other possible methods. Research currently underway is aimed at developing new techniques, including ozonation, and use of dry mist-generated hydrogen peroxide, plant extracts, and nonthermal plasma to combat antibiotic-resistant species. ${ }^{7-11}$

Atmospheric pressure nonthermal dielectric barrier discharge (DBD) plasma along with atmospheric pressure plasma jets (APPJ) with inert gases have been introduced as new, effective, and safe methods in the decontamination of living tissue. ${ }^{12-14}$ Plasma, the fourth state of matter, is a cocktail of electrons, uncharged gas molecules, atoms, free radicals, ions, and UV photons. ${ }^{15}$ Different forms of plasma can be generated using different energy sources, including lasers, heat, electrical current, and a strong electromagnetic field. In this study, DBD plasma is used, which is of particular interest in research on its possible biomedical use. ${ }^{16}$ Several studies suggest that reactive species produced in plasma, such as nitric oxide and hydroxyl radicals, have antimicrobial effects and can be useful in the decontamination of human tissue. ${ }^{17-20}$ The application of DBD plasma on human tissue is particularly promising because of its low temperature, the straightforward setup, and low electric current. ${ }^{21}$ In addition, using air as the working gas in atmospheric pressure provides a huge advantage over other plasma systems in which gas delivery systems should be used. DBD plasma treatment is a contact-free and painless procedure, causing no harm to the tissue while killing many known pathogens and also promoting wound healing. ${ }^{22}$

The current study focuses on the efficacy of DBD plasma treatment on bacteria in planktonic phase and in colonies. Bacteria were grown in vitro as planktonic cultures and in colonies as a common form of bacterial cell aggregation to compare the efficacy of DBD plasma treatment on each phase. ${ }^{23}$

The aim of this work is to study the effect of plasma in the decontamination of methicillin-resistant Staphylococcus aureus (MRSA) and Escherichia coli in planktonic cultures and in colonies in vitro.

\section{MATERIALS AND METHODS}

\section{A. Bacterial Culture and Sample Preparation}

\section{Planktonic Bacterial Culture}

To study the effect of plasma treatment on planktonic culture, one lyophilized cylinder of $E$. coli (NCTC 10538, Microbiologics) was rehydrated in super broth (SB) (Fisher 
Scientific, MA). Dehydrated pellets of MRSA strains ATCC BAA-1556, ATCC BAA1717, and ATCC 33591 (ATCC,VA), were rehydrated in brain-heart infusion (BHI) broth (Sigma-Aldrich, MO), tryptic soy broth (TSB) (Fisher Scientific, MA), and nutrient broth (Sigma-Aldrich, MO), respectively. Each culture was streaked onto an agar plate using sterile swabs and incubated at $37^{\circ} \mathrm{C}$ for 24 hours. A single colony of each strain was then transferred and inoculated in liquid broth. It was then grown overnight on a shaker platform at $37^{\circ} \mathrm{C}$. The culture was centrifuged and the supernatant was removed. The pellet was resuspended in $20 \mathrm{~mL}$ of liquid broth with $15 \%$ glycerol and transferred to microcentrifuge tubes, maintained at $-80^{\circ} \mathrm{C}$ freezer until further use.

Prior to plasma treatment, E. coli and MRSA samples were removed from the $-80^{\circ} \mathrm{C}$ freezer, thawed, and cultured in liquid broth overnight on a shaker platform at $37^{\circ} \mathrm{C}$. The cultures were diluted to obtain a cell concentration of $10^{8} \mathrm{CFU} / \mathrm{mL}$. The concentration was confirmed by a microplate reader (SpectraMax Plus 384, Molecular Devices, CA) and verified by a colony counting assay. Serial dilutions of $10^{7}, 10^{6}, 10^{5}, 10^{4}$, and $10^{3}$ $\mathrm{CFU} / \mathrm{mL}$ were prepared. $100 \mu \mathrm{L}$ of each dilution (for E. coli and all MRSA strains) were spread over agar plates using sterile spreading beads. The plates were treated with plasma for various times to study the antibacterial effect of the plasma treatment.

\section{Bacterial Colonies}

To evaluate the effect of plasma treatment on bacterial colonies, E. coli and MRSA ATCC BAA-1556 were used. This strain of MRSA is the most resistant to plasma treatment in the planktonic phase. Samples were taken out of the $-80^{\circ} \mathrm{C}$ freezer, thawed, and cultured overnight in $\mathrm{SB}$ and $\mathrm{BHI}$, respectively, on a shaker platform at $37^{\circ} \mathrm{C}$. Cultures were diluted to reach a cell concentration of $10^{8} \mathrm{CFU} / \mathrm{mL}$. These concentrations were verified with a microplate reader. Serial dilutions of $10^{3}$ and $10^{4} \mathrm{CFU} / \mathrm{mL}$ of each culture of $E$. coli and MRSA were prepared. $100 \mu \mathrm{L}$ of each dilution was spread over sterile SB and BHI agar plates, respectively, using sterile spreading beads. Then the agar plates were incubated at $37^{\circ} \mathrm{C}$ for 12 hours until the colonies had grown and were visible on the plates. The plates were then treated with plasma to study the bactericidal efficacy of the treatment.

\section{B. Dielectric Barrier Discharge Plasma Treatment}

DBD plasma was generated using a copper core electrode insulated by a Teflon hollow cylinder and a quartz dielectric barrier as the high voltage electrode. A schematic of the experimental setup is shown in Figure 1. The copper rod with 25-mm diameter was insulated with a 10-mm layer of Teflon, and a 1-mm thick quartz plate was used as the dielectric barrier. The samples were placed on a grounded plate, and the air gap between the high voltage electrode and sample surface was adjusted to $1 \mathrm{~mm}$. The direct plasma treatment was applied on a circle in the center of the sample plates that created an area with a $25-\mathrm{mm}$ diameter (core electrode diameter). This area was referred to as the plasma treated area. The applied maximum high voltage output was $10 \mathrm{kV}$ at $500 \mathrm{~Hz}$ and had a $3-\mu \mathrm{sec}$ pulse width, with a power of $0.12 \mathrm{~W} / \mathrm{cm}^{2}$.

Volume 5, Issue 1, 2015 
Agar plates inoculated with planktonic bacteria were placed on the grounded electrode, and the high voltage electrode was placed $1 \mathrm{~mm}$ from the sample surface, as shown in Figure 1. Samples were treated with plasma for 15 seconds, 30 seconds and 60 seconds, and untreated samples were used as the control. After plasma treatment, plates were incubated for 24 hours at $37^{\circ} \mathrm{C}$ for further characterization of the effect of the DBD plasma treatment. All of the experiments were performed in triplicate.

For bacterial colonies, inoculated agar plates were incubated for 12 hours to grow bacterial colonies. The agar plates were placed on the grounded electrode, and the high voltage electrode was positioned at $1 \mathrm{~mm}$ from the sample surface. Plates were treated with DBD plasma for 1, 2, 3, 4, and 5 minutes, with untreated (0 minutes) samples used as the control. Microbiological analysis and quantification of the surviving populations of bacteria were performed.

\section{Microbiological Analysis}

\section{Planktonic Bacterial Culture}

To investigate the effect of plasma treatment on planktonic cultures, plasma-treated plates were incubated and monitored daily for 72 hours to check for further bacterial growth on the plasma treated area. To investigate whether the effect of plasma treatment was bacteriostatic or bactericidal, the clear plasma-treated area of each plate was swabbed after 24 hours of incubation, using a sterile cotton swab, and streaked on another sterile plate. The cloudy, covered area was also swabbed as a control and streaked on a sterile plate. Plates were incubated for another 24 hours to analyze the effects of plasma treatment.

\section{Bacterial Colonies}

To analyze the effects of the plasma treatment on colonies, the untreated control colonies and plasma-treated colonies were monitored. After plasma treatment, plates were

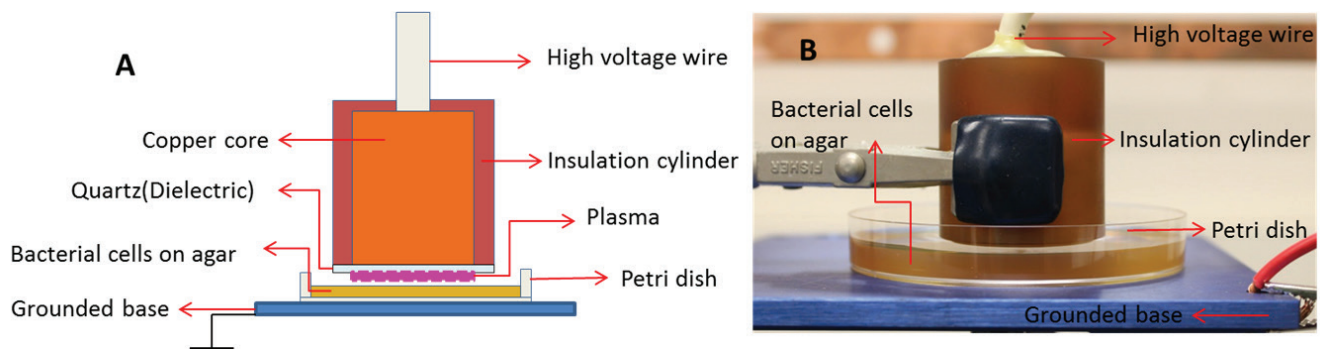

FIG. 1: A) Schematic view of the DBD plasma system. The DBD plasma system was used for the treatment of $E$. coli and MRSA cells in planktonic phase and in colonies. B) Image of DBD plasma system 
incubated for another 12 hours to observe the effect of the treatment, after which the respective sizes of treated and untreated colonies were measured and compared. For the purpose of this comparison, the diameters of the colonies were measured before plasma treatment and immediately after plasma treatment, and after 12 hours of post-treatment incubation. The measurements were done by analyzing the pictures of the samples using public domain software Image (National Institutes of Health [NIH], USA). The diameter of at least 10 colonies from each group was measured, and the values are shown in the results (Figure 6 ) as the mean \pm standard deviation.

\section{a. Quantification of the Viable CFUs within Plasma-Treated Colonies}

To determine the number of viable CFUs in samples treated with plasma for 1, 2, 3, 4, or 5 minutes and the untreated control samples, colonies were scrapped off from the agar plates using a sharp sterile needle tip and transferred to $1 \mathrm{~mL}$ of liquid media. The suspension was homogenized by pipetting up and down and then vortexing. Serial dilution was performed for the suspension, and $100 \mu \mathrm{L}$ of each dilution was inoculated on an agar plate and incubated at $37^{\circ} \mathrm{C}$ for 24 hours. After 24 hours the number of bacterial colonies was counted, and the number of viable CFUs in each colony was calculated.

\section{b. Assessment of Regrowth of Plasma-Treated Colonies in Fresh Media}

To study the regrowth of viable bacterial cells, plates were seeded with $\sim 2 \times 10^{3} \mathrm{CFU} /$ $\mathrm{mL}$ of bacteria and incubated for 12 hours. Following incubation, plates were treated with DBD plasma for 1, 2, 3, 4, or 5 minutes; untreated colonies were used as controls. Immediately after the plasma treatment, single colonies were scraped off of the plasmatreated area using a sharp sterile needle tip and transferred to $4 \mathrm{~mL}$ of liquid media. The suspension was homogenized by vortexing and incubated on a shaker platform at $37^{\circ} \mathrm{C}$. The regrowth of bacterial cells in fresh media was monitored by reading the absorbance at $600 \mathrm{~nm}$ (OD600) every 20 minutes for 140 minutes for MRSA and for 200 minutes for $E$. coli. The dynamics of the bacterial growth were measured by plotting the cell growth (absorbance) against the incubation time. Using this $\mathrm{OD}_{600}$ value and the standard curve of each bacterial species, a standardized regrowth curve of each treated colony was plotted.

\section{Penetration of DBD Plasma-Generated Nitric Oxide in Agarose Gel}

The plasma treatment was conducted at ambient environmental conditions. Studies showed that nitric oxide is likely the most well-known agent in decontamination when plasma is ignited in air. ${ }^{24,25}$ Nitric oxide introduced to the medium rapidly reacts with oxygen and produces stable metabolites of nitrate $\left(\mathrm{NO}_{3}\right)$ and nitrite $\left(\mathrm{NO}_{2}\right)$, which can be detected by Griess reaction. ${ }^{26,27}$ To study the penetration of the nitric oxide in agar after plasma treatment, a series of agar plates was prepared and seeded with $\sim 2 \times 10^{3} \mathrm{CFU} /$ $\mathrm{mL}$ of bacteria and incubated for 12 hours, after which the plates were treated with

Volume 5, Issue 1, 2015 
DBD plasma for 1, 2, 3, 4, or 5 minutes. A Griess Reagent Kit (Molecular Probes, Life Technologies, CA) was used to study the penetration of nitric oxide into agarose gel after treatment. $100 \mu \mathrm{L}$ of each reagent, R1 (sulfanilic acid) and R2 ( $N$-[1-naphthyl] ethylenediamine), was pipetted onto the plasma-treated plate, then spread and incubated for 15 minutes to allow the reagents to diffuse into the agar and for the reaction and color change to happen.

\section{E. Statistical analysis}

A minimum of three samples $(n=3)$ were used for experimental and control groups. All statistical differences were determined using the $t$-test in Microsoft Office Excel data analysis add-in with a 95\% confidence interval $(p<0.05)$. A $P$ value less than 0.05 was considered to be statistically significant. All values are reported as the mean \pm the standard deviation of the mean.

\section{RESULTS}

\section{A. The Efficacy of DBD Plasma Treatment on Planktonic Bacterial Culture}

The decontamination effect of the plasma treatment on planktonic bacteria is represented in Figure 2. The results show complete decontamination of the plasma-treated area with a surface density of $1.27 \times 10^{5} \mathrm{CFU} / \mathrm{cm}^{2}$ for MRSA strains ATCC BAA-1556, ATCC BAA-1717, and ATCC 33591 after 60 seconds of treatment, and for E. coli NCTC 10538 after 30 seconds of treatment. The plasma-treated area is defined as a circle with a $25-\mathrm{mm}$ diameter on which the plasma is applied directly. After the center of each plate (seeded with $10^{8} \mathrm{CFU} / \mathrm{mL}$ ) was treated with plasma for 60 seconds, complete decontamination on the plasma treated area was observed, and after 24 hours of incubation at $37^{\circ} \mathrm{C}$, no bacteria growth took place on the plasma-treated area.

To study whether the effect of plasma treatment is bactericidal or bacteriostatic, the clear plasma treated area was swabbed and streaked on a sterile agar plate. Plates were monitored for 72 hours, and after this period of incubation, no bacteria regrowth was observed. As a control, the untreated area was also swabbed and streaked on a new agar plate; after 24 hours of incubation bacterial growth could be seen (Fig. 3, B).

\section{B. The Efficacy of DBD Plasma Treatment on Bacterial Colonies}

The decontamination effect of the plasma treatment on bacterial colonies is represented in Figures 4 and 5. The black circles on the plates show the plasma-treated area. The small marks on the plasma-treated area are where colonies were scraped off for further characterization. As shown, E. coli colonies stopped growing after 5 minutes of plasma treatment. For MRSA strain ATCC BAA-1556, colony size is approximately unchanged after 24 hours of incubation at $37^{\circ} \mathrm{C}$. Colonies from test samples with shorter treatment times did grow after 24 hours of post-treatment incubation at $37^{\circ} \mathrm{C}$. However, growth 


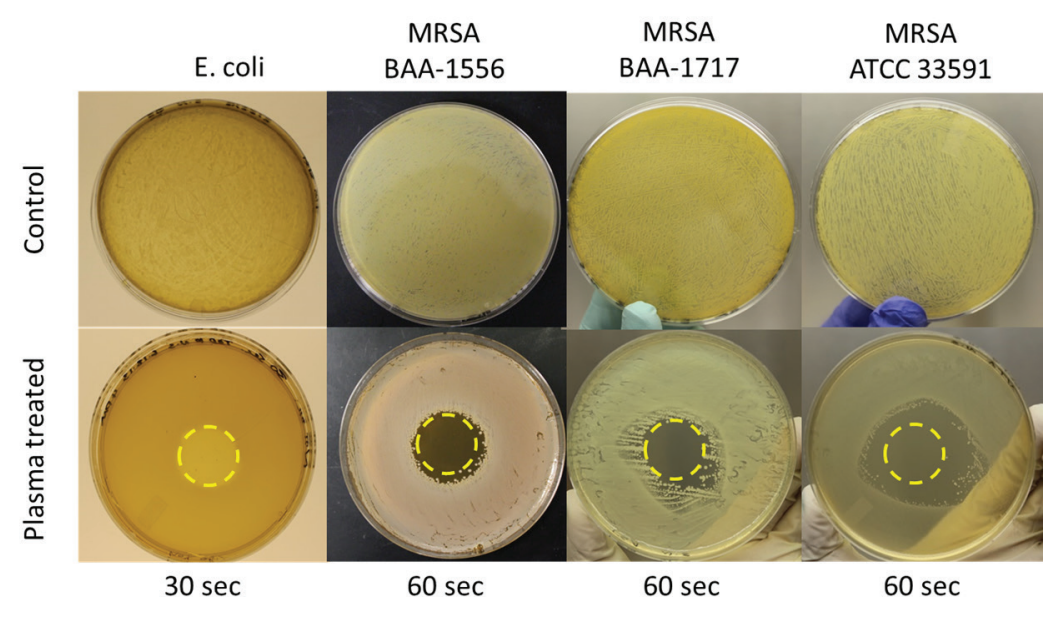

FIG. 2: Plates seeded with planktonic bacterial culture with the concentrations of $\sim 10^{8} \mathrm{CFU} / \mathrm{mL}$ creating a bacterial surface density of $\sim 10^{5} \mathrm{CFU} / \mathrm{cm}^{2}$. Complete sterilization on the plasma-treated area (dashed circles) is achieved for $E$. coli after 30 seconds and for MRSA ATCC BAA-1556, MRSAATCC BAA-1717, and MRSAATCC 33591 after 60 seconds of plasma treatment

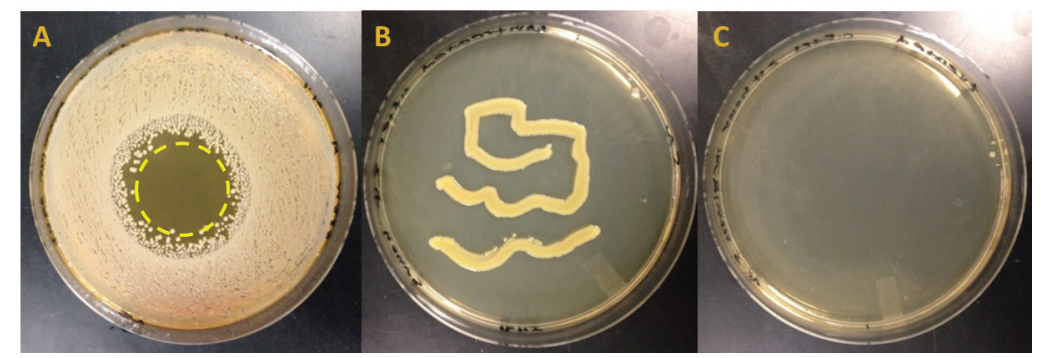

FIG. 3: A) MRSA (ATCC BAA-1556) culture on agar after treatment and 24 hours of post-treatment incubation. The plasma-treated area that (dashed circle) is completely clear. B) Untreated area was swabbed and streaked on a sterile agar plate. After 24 hours of post-treatment incubation, bacterial growth is present. C) Plasma treated area is swabbed and streaked on a sterile agar plate. No bacterial regrowth was observed after 3 days of post-treatment incubation.

in the treated colonies was less substantial than in the untreated colonies after 12 hours of incubation, and as treatment time increased, the level of growth in the colonies decreased, when measured after 24 hours of post-treatment incubation at $37^{\circ} \mathrm{C}$. Figure 6 shows the final diameter of the $E$. coli colonies incubated for 12 hours following various lengths of plasma treatment.

Volume 5, Issue 1, 2015 

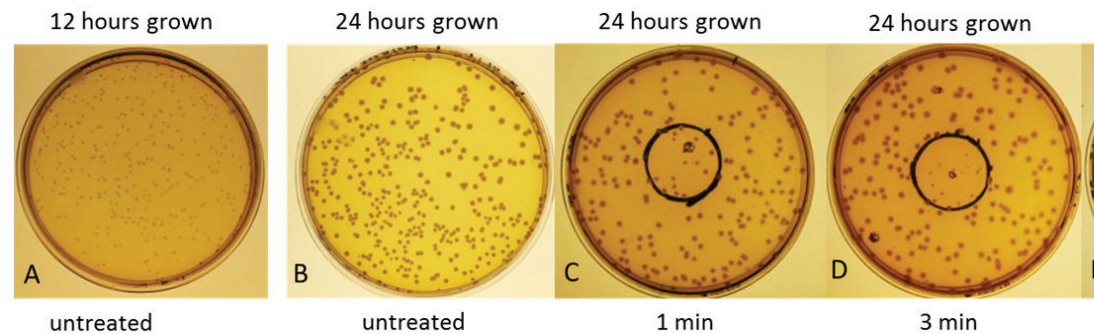

24 hours grown

FIG. 4: A) E. coli colonies incubated for 12 hours. After colonies are grown for 12 hours, they are plasma treated for 1 to 5 minutes to observe the effect on the colony growth process. B, C, D,E) E. coli colonies after 1, 3, and 5 minutes of plasma treatment and an additional 12 hours of post-treatment incubation. The black circles show the plasma treatment area. Results demonstrated that the colonies in the plasma-treated area are smaller than untreated colonies that had gone through the normal growth process.

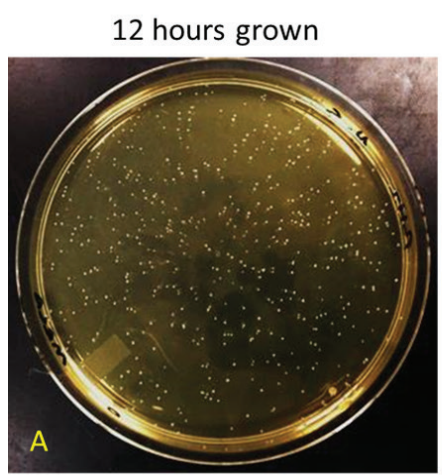

untreated

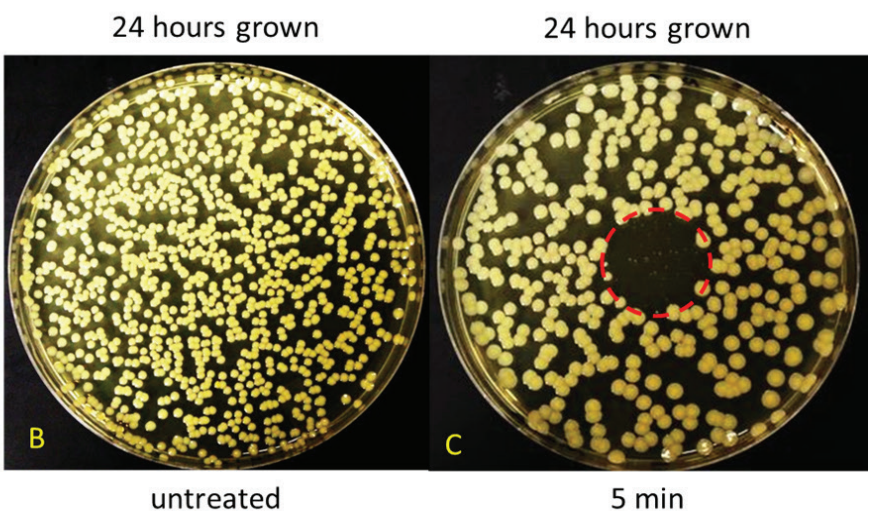

untreated
$5 \mathrm{~min}$

FIG. 5: A) MRSA colonies incubated for 12 hours. B) MRSA colonies after 5 minutes of plasma treatment and an additional 12 hours of pos-treatment incubation. The colonies in the plasma-treated area (dashed circles) are smaller than the colonies outside of the plasma-treated area.

\section{Quantification of the Viable CFUs in Plasma-Treated Colonies}

Colonies were treated on the plates for 1 to 5 minutes; the control was untreated. A portion of each sample was scraped off and transferred to $1 \mathrm{~mL}$ of fresh media. Serial dilution was performed and dilutions were plated. Colonies were counted after 24 hours, and the number of viable CFUs was calculated for each colony. Samples were prepared in triplicate; at least three colonies from each plate were removed and the number of CFUs in each was calculated. The results for E. coli and MRSA colonies are shown in Figures 7 and 8 respectively. The values are mean \pm standard deviation of at least 


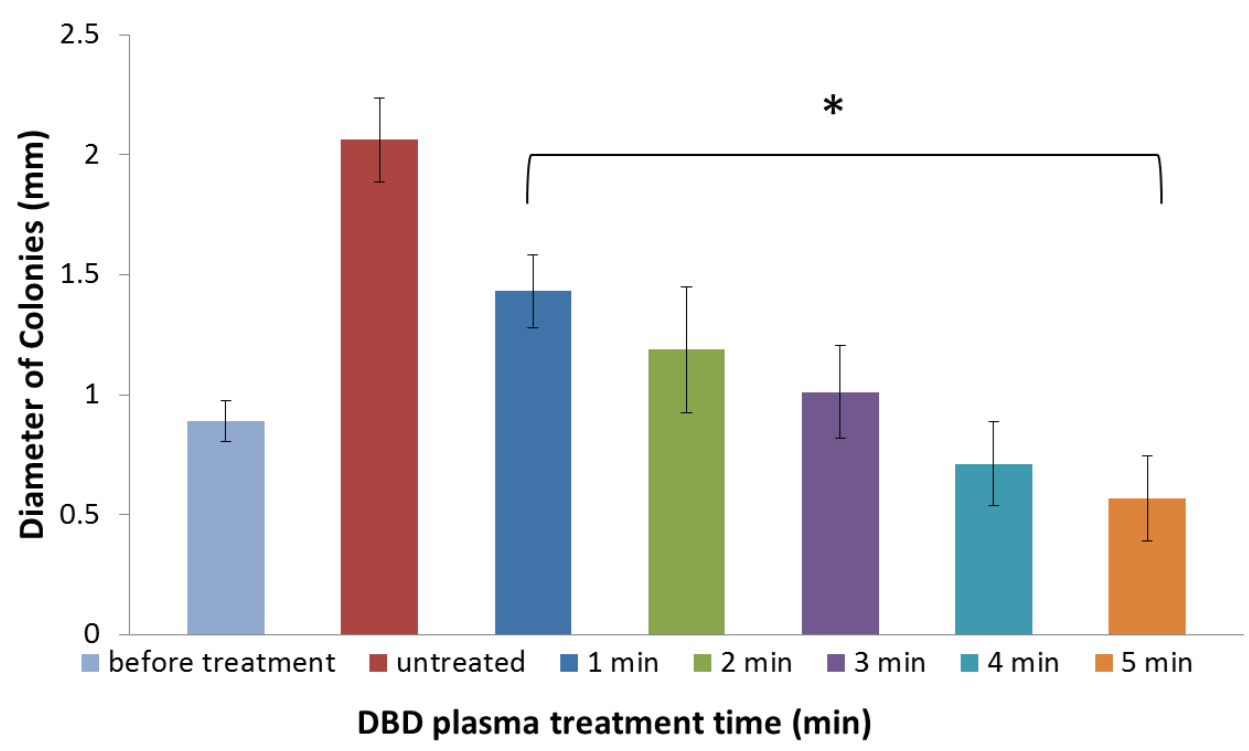

FIG. 6: The first bar represents the diameter of the colonies before plasma treatment. The rest of the bars show the final diameter of E. coli colonies after additional 12 Hours of post-treatment incubation. The results are expressed as mean \pm standard deviation of at least 10 samples. Asterisks $\left(^{*}\right)$ denote statistical differences from the untreated group. $(P<0.0001[t$-test $])$

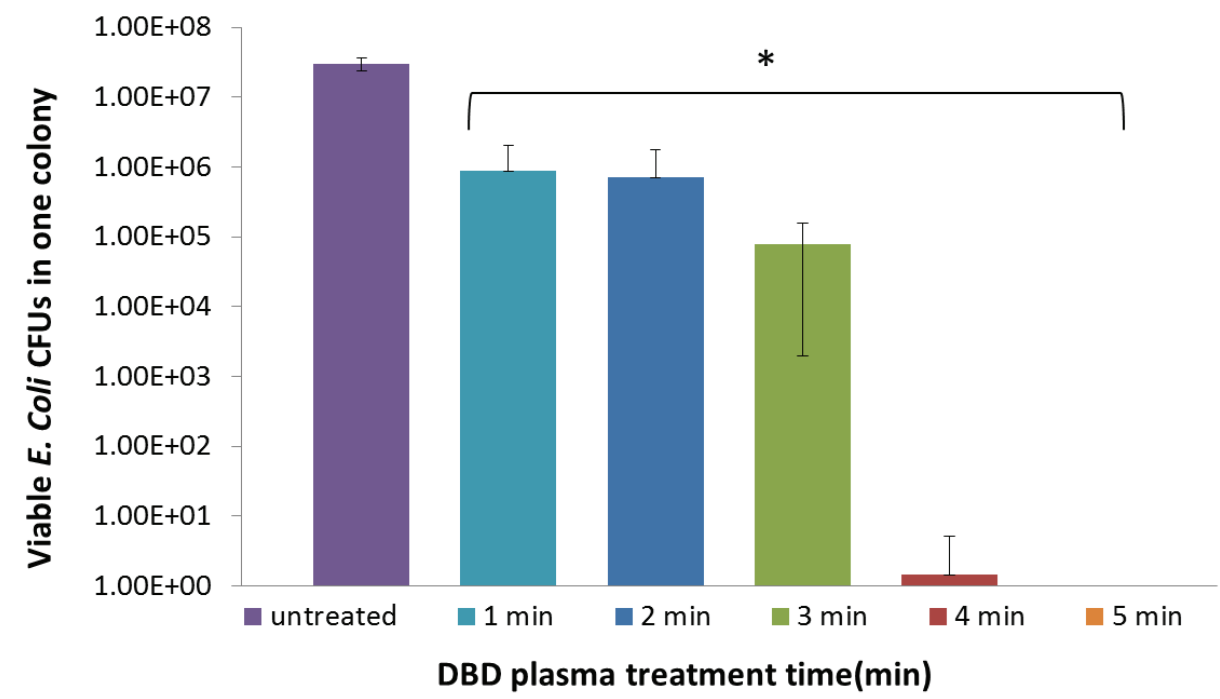

FIG. 7: Number of viable CFUs of $E$. coli in each colony after various treatment times. The results are expressed as mean \pm standard deviation of at least 7 samples. Asterisks $\left(^{*}\right)$ denote statistical differences from the untreated group. $(P<0.0001$ (t-test) $)$ 


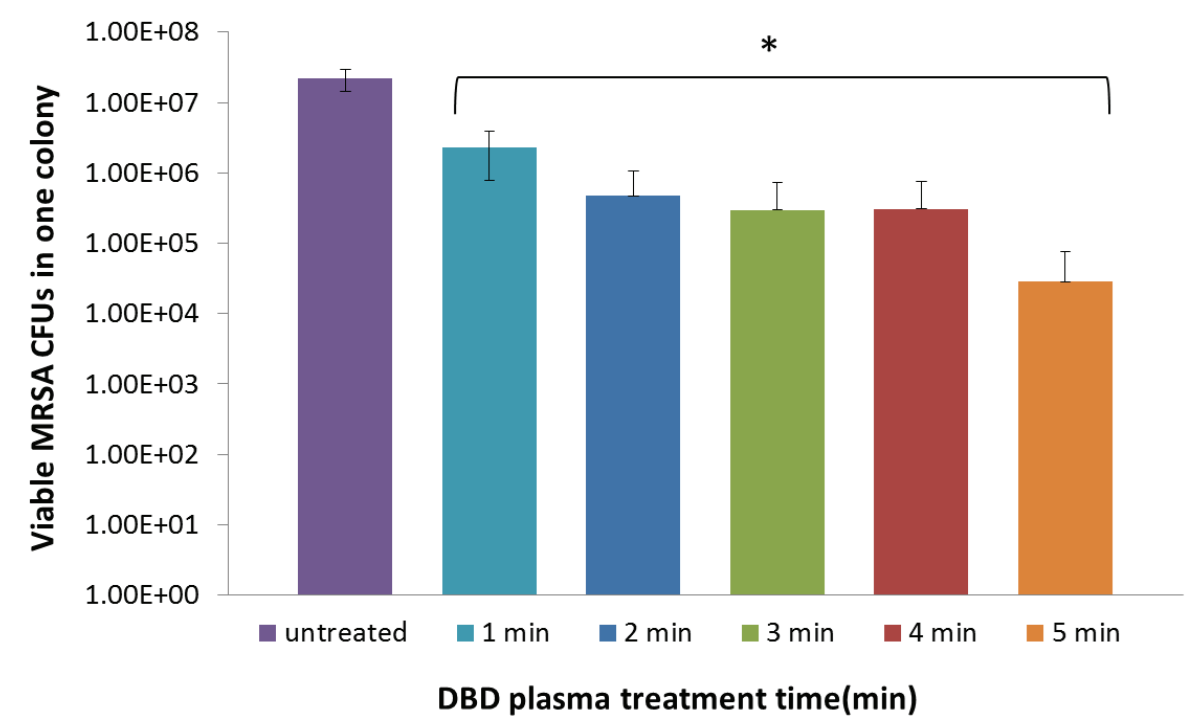

FIG. 8: Number of viable CFUs of MRSA in each colony after various treatment times. The results are expressed as mean \pm standard deviation of at least 7 samples. Asterisks $\left({ }^{*}\right)$ denote statistical differences from the untreated group. $(P<0.0001$ [t-test] $)$

10 samples. Results show $7 \log _{10}$ reductions for $E$. coli colonies after 5 minutes of plasma treatment. For MRSA colonies, 3 to $7 \log _{10}$ reductions were observed after 5 minutes of plasma treatment. Longer treatment time is needed to achieve complete decontamination for MRSA colonies.

\section{Assessment of Regrowth of Plasma Treated Colonies in Fresh Media}

Colonies were cut and removed from the agar plate after treatment, transferred into $4 \mathrm{~mL}$ of liquid media, and incubated on a shaker platform at $37^{\circ} \mathrm{C}$. The growth was monitored. The influence of the plasma treatment on the regrowth of treated E. coli and MRSA colonies is shown in Figures 9 and 10.

\section{Penetration of DBD Plasma-Generated Nitric Oxide into Agarose Gel}

The results of Griess assays on the plasma-treated area after different treatment times are shown in Figure 11. Color change indicates that nitric oxide diffused into the gel and that nitrite is present in the area. The nitric oxide diffusion area and color intensity are greater with increasing plasma treatment time, as shown in Figure 11 The nitric oxide diffusion area was measured using a threshold color plugin from the public domain software ImageJ (National Institutes of Health (NIH), USA). Images were converted to 8-bit grayscale, and their threshold was adjusted so that the colored area was distinct. The picture was then analyzed, and the diffusion change area was measured. In samples 


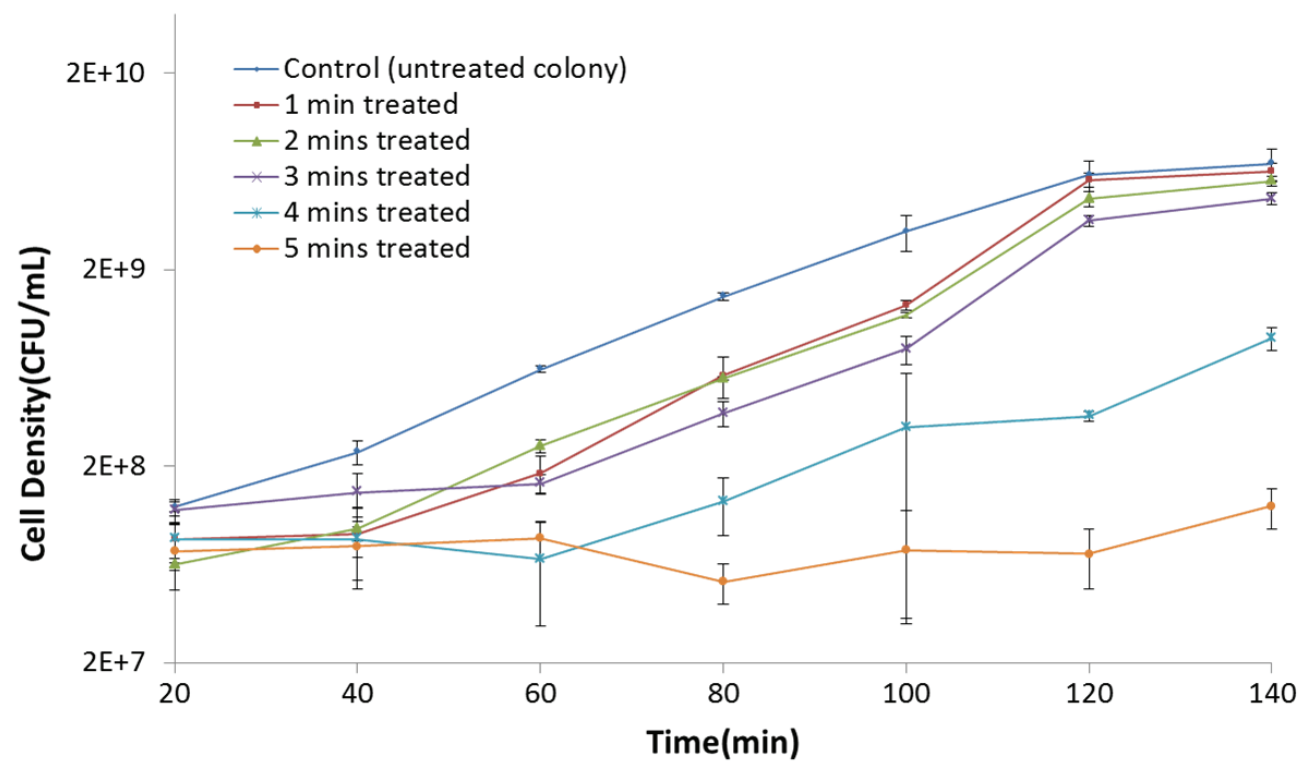

FIG. 9: Regrowth of E. coli Colonies in SB Media after Plasma Treatment. Treated colonies were transferred to fresh media, and the regrowth was monitored by reading the $\mathrm{OD}_{600}$ of the cultures every 20 minutes. Results are expressed as mean \pm standard deviation of four samples.

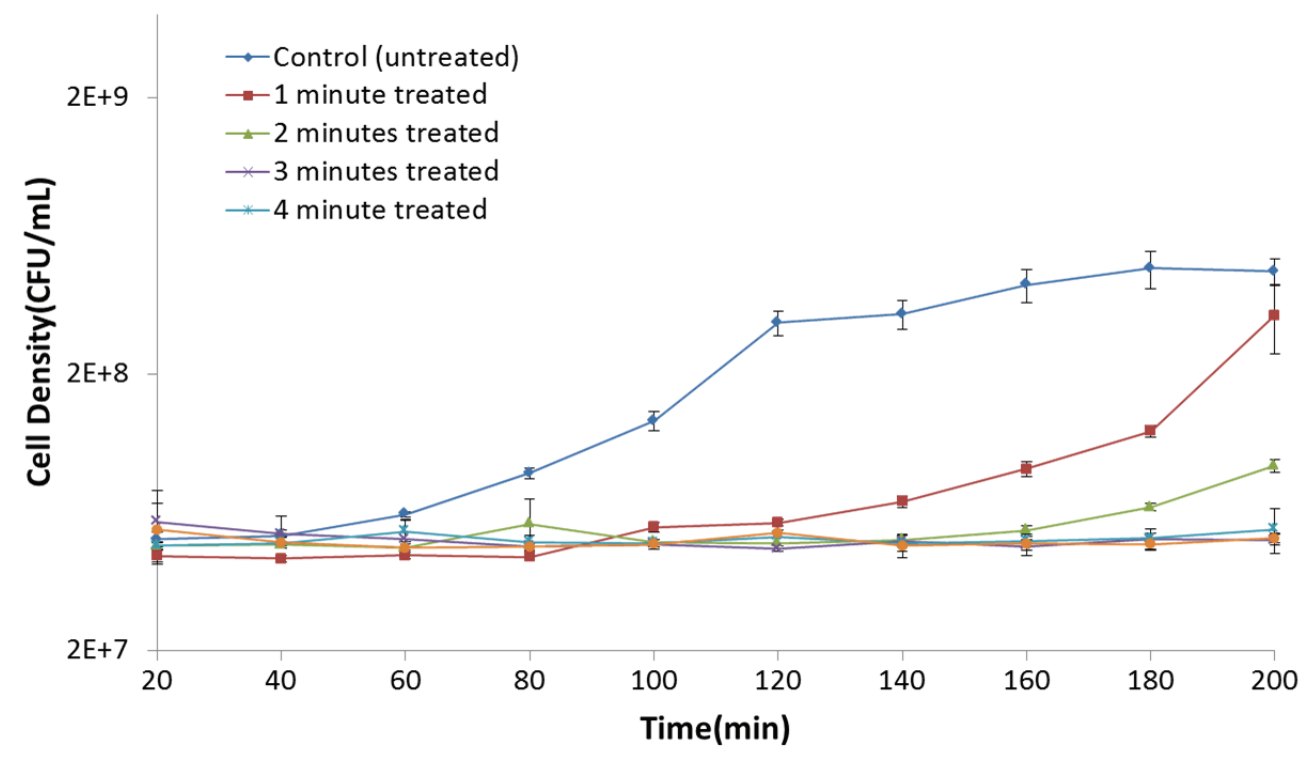

FIG. 10: Regrowth of MRSA Colonies in BHI Media after Plasma Treatment. Treated colonies were transferred to fresh media, and the regrowth was monitored by reading the $\mathrm{OD}_{600}$ of the cultures every 20 minutes. Results are expressed as mean \pm standard deviation of four samples.

Volume 5, Issue 1, 2015 

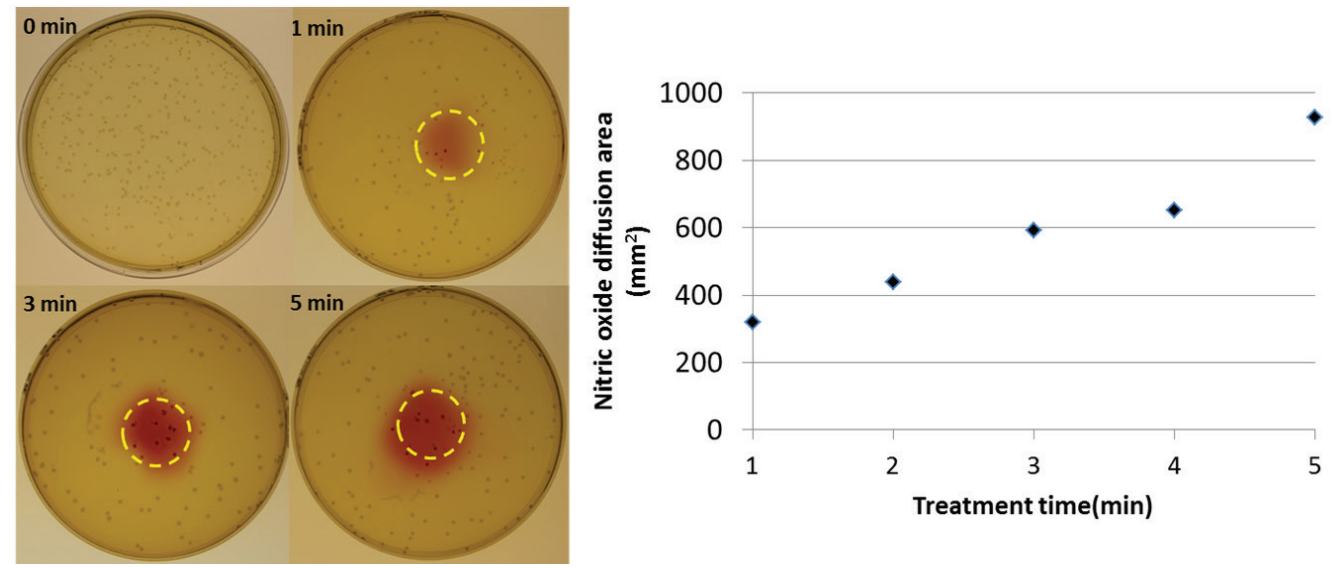

FIG. 11: Diffusion of nitric oxide in agarose gel is detected with the Griess assay after 1, 2, 3,4 , or 5 minutes of plasma treatment. The plasma-treated area is specified with the dashed circles. The diffusion area grows bigger with increasing the treatment time. The areas were measured using ImageJ software.

that were treated with plasma for 3 minutes, the diffusion area was larger than the area directly treated with plasma, which is equal to core electrode area.

\section{DISCUSSION}

In this study, the decontamination efficacy of DBD plasma treatment on E. coli and MRSA was investigated in a their planktonic phase and in colonies. Results demonstrated that DBD plasma can kill antibiotic-resistant species.

Plasma is a cocktail of agents capable of decontamination. It contains a high concentration of reactive oxygen and nitrogen species, such as nitric oxide, hydrogen peroxide, and hydroxyl radicals. ${ }^{28}$ In addition to the strong electric field between the closely spaced electrodes, two central mechanisms have been implicated in decontamination by plasma: the reactive species that are generated in the plasma can deactivate the bacterial cells either through direct physical bombardment of the membrane or by diffusing through the bacterial cell membrane and reacting with the membrane. ${ }^{29,30}$

The DBD plasma system was created and electrically characterized. Further the DBD plasma was used to treat E. coli and MRSA in the planktonic phase and in colonies for various treatment times. Results confirmed that DBD plasma treatment is decontaminating all the strains in planktonic phase and is damaging to the bacteria in colonies. Finally, we demonstrated that nitric oxide is diffusing more in the plasma-treated agar plates with increasing the treatment times.

The planktonic cultures of MRSA and E. coli were treated for various times by DBD plasma. After 60 seconds of plasma treatment of plates seeded with planktonic culture of all strains of MRSA (having the concentration of $10^{8} \mathrm{CFU} / \mathrm{mL}$ ), complete decontamina- 
tion on the plasma treated area was observed. The treated area had a bacterial surface density of $1.27 \times 10^{5} \mathrm{CFU} / \mathrm{cm}^{2}$ (Figure 2). No bacterial growth occurred on the plasmatreated area after 24 hours of incubation. This happened for $E$. coli cells after 30 seconds of treatment (Figure 2). These results indicate that MRSA strains are more resistant to plasma treatment than E. coli. By decreasing the bacterial concentration and increasing the treatment time, the size of clear decontaminated area increases and will eventually expand beyond the size of the area that was directly treated with plasma, which is equal to the core electrode area. This suggests that the reactive species diffuse beyond the electrode area. Therefore, the effect of the reactive species in the decontamination process is chemical and not just related to the physical bombardment of the bacterial cells with ions or other charged particles. .

Bactericidal drugs kill bacteria directly, whereas bacteriostatic drugs simply inhibit bacterial growth. To test the bactericidal effect of the plasma treatment, plasma-treated bacterial cultures were transferred from the plasma-treated area that is decontaminated and clear, to a new agar plate. This would permit growth of any bacteria still present in the absence of antibacterial agents introduced to the agar plate following the plasma treatment. The agar plates were monitored for a few days, and no bacterial growth was observed (Figure 3). This suggests that the plasma treatment may have a bactericidal effect on the bacterial cells during the decontamination process.

The minimum time needed to completely inactivate MRSA in planktonic phase was 60 seconds, so this time was chosen as the minimum time to treat the colonies. Results demonstrated that longer treatment times are required to decontaminate the bacterial cells in colonies than in planktonic phase. This might occur because cell densities are higher in colonies. In addition, bacterial cells in colonies are encased in a polysaccharide matrix and as a result may be more resistant to the treatment. Colonies were treated for $1,2,3,4$, or 5 minutes. For all the treatment times, the plasma-treated $E$. coli colonies exhibited less growth at 24 hours after plasma treatment compared to untreated colonies (Figure 4). Also, the final diameter of the plasma-treated MRSA colonies at 24 hours post-treatment incubation are less than the diameter of untreated colonies (Figure 5).The final diameter of the $E$. coli colonies incubated for an additional 12 hours post-plasma treatment decreased as the treatment time increased (Figure 6). The diameter of the E. coli colonies before treatment was $0.88 \pm 0.13 \mathrm{~mm}$, which is slightly greater than the diameter of colonies after 5 minutes of treatment followed by 12-hour post-treatment incubation. This could represent some level of shrinkage in colonies due to bacterial cell damage after 5 minutes of plasma treatment.

Results demonstrated that plasma treatment significantly reduced the number of viable CFUs in colonies after 1 minute of plasma treatment (Figures 7 and 8), and monitoring the regrowth of treated colonies in liquid media justifies this finding. After quantification of the number of viable CFUs in plasma treated colonies, results indicate that for $E$. coli colonies 4 and 5 minutes of plasma treatment is resulting in $7 \log _{10}$ reductions in all the colonies in the plasma treated area (Figure 7). For MRSA colonies more treatment time is needed for decontamination of all the colonies in the plasma treated

Volume 5, Issue 1, 2015 
area as the colonies show more resistance to the plasma treatment compare to E. coli colonies. The results show a range of $2-7 \log _{10}$ reduction in the number of viable CFUs from the areas treated with plasma for 5 minutes (Figure 8). This means that the effect of plasma treatment is not distributed uniformly on all the colonies, and longer treatments are needed to induce equal levels of decontamination in all the MRSA colonies in the plasma-treated area.

The length of the lag phase is dependent on a variety of factors, including change in physiology, nutrients, and time to recover from shock in the transfer, which we assume should be present for all the transferred colonies. ${ }^{31}$ Time needed for recovery from damage and the size of the inoculum, which means the number of cells in each colony that are dividing normally, are two other important factors influencing the length of the lag phase. ${ }^{32}$ Growth curves for colonies treated for various times are showing that colonies treated for longer times have a longer lag phase, which could mean that more damage is induced in the colonies with increasing treatment times. No regrowth of 4- and 5-minute treated E. coli colonies is observed in fresh media after 200 minutes incubation on a shaker platform at $37^{\circ} \mathrm{C}$ (Figure 9). However, monitoring the regrowth of MRSA colonies in fresh media for 140 minutes confirmed that extended treatment time is required to deactivate all the CFUs in MRSA colonies (Figure 10).

With longer treatment times, reactive species can reach beyond the plasma-treated area in gaseous phase as well as penetrate more into the agar gel along the surface and at deeper levels. Figure 11 represents the increasing diffusion of nitric oxide in the agar as treatment time increases. It can be seen that the nitric oxide diffusion area becomes broader as treatment time increases. This indicates that with increased treatment time, more nitric oxide penetrates into the solid agar, and this can increase the area of decontamination.

\section{CONCLUSION}

Antibiotic resistance in $S$. aureus, one of the most common musculoskeletal pathogens, is a serious problem. New treatment methods are needed to combat these pathogens. The effect of cold plasma treatment on the decontamination of MRSA and E. coli in planktonic cultures and colonies was studied. Cold plasma treatment is a bactericidal treatment and is an effective way for inactivating $E$. coli and MRSA strains ATCC BAA1556, ATCC BAA-1717, and ATCC 33591 in planktonic phase and in colonies. Complete sterilization occurs after 60 seconds for all the strains in planktonic phase. Longer treatment times are needed to observe a uniform distribution of treatment effect for colonies on the plasma-treated area. For a given strain, longer treatment time is needed to completely inactivate bacteria in colonies than in planktonic phase. With longer treatment times, the reactive species, such as nitric oxide, diffuse more into the agar and can widen the area of decontamination. 


\section{ACKNOWLEDGMENT}

The authors would like to thank Dr. Eda Yildirim-Ayan, Department of Bioengineering, University of Toledo, for sharing her scientific insight and knowledge. The excellent technical assistance of Tamara L. Phares is also gratefully acknowledged.

\section{REFERENCES}

1. Gillaspy AF, Hickmon SG, Skinner RA, Thomas JR, Nelson CL, Smeltzer MS. Role of the accessory gene regulator (agr) in pathogenesis of staphylococcal osteomyelitis. Infect Immun. 1995;63:3373-80.

2. Burnett MW, Bass JW, Cook BA. Etiology of osteomyelitis complicating sickle cell disease. Pediatrics. 1998;101(2):296-7.

3. Tiemann AH, Hofmann GO. Principles of the therapy of bone infections in adult extremities. Strategies Trauma Limb Reconstr. 2009;4(2):57-64.

4. DeLeo FR, Chambers HF. Reemergence of antibiotic-resistant Staphylococcus aureus in the genomics era. J Clin Invest. 2009;119(9):2464-74.

5. Bowler PG, Duerden BI, Armstrong DG. Wound microbiology and associated approaches to wound management. Clin Microbiol Rev. 2001;14(2):244-69.

6. Wright JB, Lam K, Burrell RE. Wound management in an era of increasing bacterial antibiotic resistance: a role for topical silver treatment. Am J Infect Control. 1998;26(6):572-77.

7. Lüddeke F, Heß S, Gallert C, Winter J, Güde H, Löffler H. Removal of total and antibiotic resistant bacteria in advanced wastewater treatment by ozonation in combination with different filtering techniques. Water Res. 2015;69:243-51.

8. Valle Jr DR, Andrade JI, Puzon JJM, Cabrera EC, Rivera WL. Antibacterial activities of ethanol extracts of Philippine medicinal plants against multidrug-resistant bacteria. Asian Pac J Trop Biomed. 2015;5(7):532-40.

9. Cotter JJ, Maguire P, Soberon F, Daniels S, O'Gara JP, Casey E. Disinfection of meticillinresistant Staphylococcus aureus and Staphylococcus epidermidis biofilms using a remote non-thermal gas plasma. J Hosp Infect. 2011;78:204-7.

10. Bartels MD, Kristoffersen K, Slotsbjerg T, Rohde SM, Lundgren B, Westh H. Environmental meticillin-resistant Staphylococcus aureus (MRSA) disinfection using dry-mist-generated hydrogen peroxide. J Hosp Infect. 2008;70:35-41.

11. Lee KY, Park BJ, Lee DH, Lee IS, Hyun SO, Chung KH, Park JC. Sterilization of Escherichia coli and MRSA using microwave-induced argon plasma at atmospheric pressure. Surf Coat Tech. 2005;193:35-8.

12. Scholtz V, Pazlarová J, Soušková H, Khun J, Julák J. Nonthermal plasma — A tool for decontamination and disinfection. Biotechnol Adv. 2015;33(6):1108-19.

13. Weltmann KD, Kindel E, von Woedtke T, Hähnel M, Stieber M, Brandenburg R. Atmospheric-pressure plasma sources: prospective tools for plasma medicine. Pure Appl Chem. 2010;82(6):1223-37.

14. Weltmann KD, Polak M, Masur K, von Woedtke T, Winter J, Reuter S. Plasma processes and plasma sources in medicine. Contrib Plasma Phys. 2012;52(7);644-54.

Volume 5, Issue 1, 2015 
15. Heinlin J, Morfill G, Landthaler M, Stolz W, Isbary G, Zimmermann JL, Shimizu T, Karrer S. Plasma medicine: possible applications in dermatology. J Dtsch Dermatol Ges. 2010;8(12):968-76.

16. Kim YJ, Jin S, Han GH, Kwon GC, Choi JJ, Choi EH, Uhm HS, Cho G. Plasma apparatuses for biomedical applications. IEEE Trans Plasma Sci. 2015;43(4):944 -50.

17. Ma DG, Fang FC. NO inhibitions: antimicrobial properties of nitric oxide. Clin Infect Dis. 1995;21(Suppl 2):S162-S165.

18. Moncada S, Palmer RM, Higgs E. Nitric oxide: physiology, pathophysiology, and pharmacology. Pharmacol Rev. 1991;43(2):109-34.

19. Liou JW, Hung YJ, Yang CH, Chen YC. The antimicrobial activity of gramicidin A is associated with hydroxyl radical formation. PLoS One. 2015;10(1):e0117065.

20. Jayaprakasha GK, Selvi T, Sakariah KK. Antibacterial and antioxidant activities of grape (Vitis vinifera) seed extracts. Food Res Int. 2003;117-22.

21. Rajasekaran P, Bibinov N, Awakowicz P. Characterization of dielectric barrier discharge in air applying current measurement, numerical simulation and emission spectroscopy. Meas Sci Technol. 2012;085605.

22. Isbary G, Stolz W, Shimizu T, Monetti R, Bunk W, Schmidt HU, Morfill GE, Klämpfl TG, Steffes B, Thomas HM, Heinlin J, Karrer S, Landthaler M, Zimmermann JH. Cold atmospheric argon plasma treatment may accelerate wound healing in chronic wounds: results of an open retrospective randomized controlled study in vivo. Clin Plasma Med. 2013;1(2) $25-30$.

23. Mikkelsen H, Duck Z, Lilley KS, Welch M. Interrelationships between colonies, biofilms, and planktonic cells of Pseudomonas aeruginosa. J Bacteriol. 2007;189(6):2411-6.

24. Schairer DO, Chouake JS, Nosanchuk JD, Friedman AJ. The potential of nitric oxide releasing therapies as antimicrobial agents. Virulence. 2012;3:271-9.

25. Kühn S, Bibinov N, Gesche R, Awakowicz P. Non-thermal atmospheric pressure HF plasma source: generation of nitric oxide and ozone for bio-medical applications. Plasma Sources Sci Technol. 2009;19(1):015013.

26. Xiao ZS, Quarles LD, Chen QQ, Yu YH, Qu XP, Jiang CH, Deng HW, Li YJ, Zhou $\mathrm{HH}$. Effect of asymmetric dimethylarginine on osteoblastic differentiation. Kidney Int. 2001;60(5):1699-704.

27. Pan W, Quarles LD, Song LH, Yu YH, Jiao C, Tang HB, Jiang CH, Deng HW, Li YJ, Zhou $\mathrm{HH}$, Xiao ZS. Genistein stimulates the osteoblastic differentiation via NO/cGMP in bone marrow culture. J Cell Biochem. 2005;94(2):307-16.

28. Kogelschatz U. Dielectric-barrier discharges: their history, discharge physics, and industrial applications. Plasma Chem Plasma P. 2003;23(1):1-46 .

29. Liu F, Sun P, Bai N, Tian Y, Zhou H, Wei S, Zhou Y, Zhang J, Zhu W, Becker K, Fang J. Inactivation of bacteria in an aqueous environment by a direct-current, cold-atmosphericpressure air plasma microjet. Plasma Processes Polym. 2009;7(3-4):231-6.

30. Dobrynin D, Fridman G, Friedman G, Fridman A. Physical and biological mechanisms of direct plasma interaction with living tissue. New J Phys. 2009;11:115020.

31. Rolfe MD, Rice CJ, Lucchini S, Pin C, Thompson A, Cameron ADS, Alston M, Stringer MF, Betts RP, Baranyi J, Peck MW, Hinton JCD. Lag phase is a distinct growth phase that prepares bacteria for exponential growth and involves transient metal accumulation. J Bacteriol. 2012;194(3):686-701.

32. Penfold WJ. On the nature of bacterial lag. J Hyg (Lond). 1914;14:215-41. 